\section{Reactions to nuclear anti-analogue states}

\author{
from P. E. Hodgson
}

REACTIONS to isobaric analogue states have been extensively studied ever since their discovery by Anderson and Wong in 1962, and have yielded much useful information on nuclear structure and on the nucleon optical potential (Nature, 247, 179; 1974). More recently, it has proved possible to detect reactions to anti-analogue states, and analyses of their cross sections are giving interesting results.

The simplest reaction to these states is the $(p, n)$ reaction, that adds a proton and removes a neutron from the ground state of the target nucleus. The final nucleus thus has the same number of nucleons as the target nucleus and essentially the same mass; this is why the final states are called 'isobaric'. At the same time the charge has been increased by one, and the ways of doing this can most easily be described using the isobaric spin formalism, which is exactly similar to the corresponding formalism for ordinary spin. Each nuclear state has an isobaric spin, with three components like a spin vector. The third component $T_{3}$ is directly related to the nuclear charge, $\mathrm{T}_{3}=\frac{1}{2}(N-Z)$, where $N$ and $Z$ are the numbers of neutrons and protons in the nucleus. All the states of a nucleus thus have the same value of $T_{3}$, but they can have any total isobaric spin equal to or greater than $T_{3}$, since no vector can be shorter than any one of its components.

The ground states of nuclei nearly always have $T=\mathrm{T}_{3}$, so when we increase the charge by one by a $(p, n)$ reaction we reduce $T_{3}$ by one, since by convention a neutron has $T_{3}=\frac{1}{2}$ and a proton has $T_{3}=-\frac{1}{2}$. The final states can thus have a total isobaric spin of $T$ or $T-1$. The state with isobaric spin $T$ is the isobaric analogue state, and apart from small electromagnetic effects it has exactly the same structure as the target ground state, except of course for the reduced charge. All the nucleons are coupled together in the same way, and we can say that the only difference is that one isobaric spin vector has been flipped so that its third component is $-\frac{1}{2}$ instead of $\frac{1}{2}$. Quantum mechanically the states have a very high overlap, and so reactions from one to the other take place easily, and it is their high cross sections that enable them to be detected even though many other reactions were also taking place.

The anti-analogue state with isobaric spin $T-1$ is just the opposite; quantum mechanically its structure is nearly orthogonal to that of the ground state of the target. Reactions to this state are thus very difficult, and can only take place at all because of small differences in structure from complete orthogonality. Unlike the analogue state reactions, the anti-analogue state reactions are sensitive to small components of the wavefunctions, and they are thus difficult to describe theoretically as well as being difficult to detect due to their low cross sections.

Early work on anti-analogue state reactions used the $\left({ }^{3} \mathrm{He}, \mathrm{t}\right)$ reaction, which also has the net effect of adding a proton and removing a neutron. Calculations of the cross section of the reaction on ${ }^{40} \mathrm{Ar}$ by Schaeffer and Bertsch (Phys. Lett., 38B, 159; 1972) showed that the direct component can only account for a very small fraction of the observed cross section, but that a reasonable fit can be obtained by assuming that the reaction takes place mainly by a two-step process in which a $\left({ }^{3} \mathrm{He}, \alpha\right)$ reaction is rapidly followed by a $(\alpha, t)$ reaction. Similar results were obtained for the $\left({ }^{3} \mathrm{He}, \mathrm{t}\right)$ reaction on ${ }^{48} \mathrm{Ca}$.

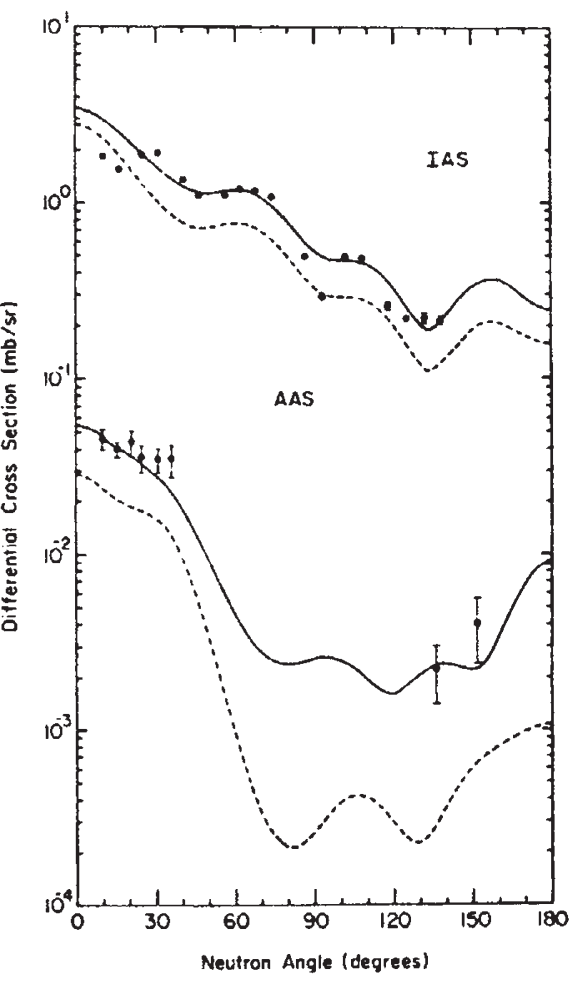

Differential cross sections of the ${ }^{40} \mathrm{Ar}(\mathrm{p}, \mathrm{n})^{40} \mathrm{~K}$ reaction at $24 \mathrm{MeV}$ to the isobaric analogue and antianalogue states compared with distorted wave calculations assuming that the reactions take place in one step. The dashed curves show the result of the direct transition only and the solid curves the result of including the knock-on exchange contributions. The isobaric analogue state cross sections were obtained by Bentley and colleagues (Phys. Rev. Lett., 27, 1081; 1971).
Studies have now been made of the $(p, n)$ reaction to the anti-analogue state of ${ }^{40} \mathrm{Ar}$ by Galonsky and colleagues of Michigan State University (Phys. Rev. Lett., 35, 1208; 1975). They used $24 \mathrm{MeV}$ protons, and were able to detect the very small cross sections of the anti-analogue state reaction using a large liquid scintillator and a time-of-flight spectrometer. As shown in the figure, these cross sections are only about $1 \%$ of the corresponding analogue state ones.

They analysed these data using the distorted wave theory assuming that the reaction takes place on one step, and including both the direct and the knock-on exchange contributions. The dashed curves in the figure are for the direct process only and the solid curves show the result of including exchange. The parameters of the distorting potentials and of the effective nucleonnucleon interaction were all taken from previous work.

It is clear from the figure that this calculation gives cross sections in very good agreement with the experimental data, showing that at least in this case it is not necessary to take into account the possibility of two-step reactions in which the incident and outgoing particles are composite, for then the exchange processes are expected to be more likely than the direct.

The relative probabilities of one-step and multi-step processes is a very interesting question, and it is likely that further studies of reactions to antianalogue state will provide important information. They should also tell us more about the smaller components of the nuclear wavefunctions.

\section{Mammalian DNA repair}

\section{from a Correspondent}

The Second International Workshop on DNA Repair Mechanisms in Mammalian Cells was held at Nordwijkerhout, the Netherlands, on May 2-5.

AlL cellular systems that have been examined possess repair mechanisms for dealing with lesions introduced into DNA. The general purpose of investigations on mammalian DNA repair and of the Second International Workshop on DNA Repair Mechanisms in Mammalian Cells has been to understand how damage in cellular DNA is recognised and removed-or alternatively bypassed-and the effect of the processes involved (that is, mutation or cancer). 